\title{
Rare-event Simulation for Stochastic Korteweg-de Vries Equation
}

\author{
Gongjun $\mathrm{Xu}^{*}$ Guang $\operatorname{Lin}^{\dagger}$ and Jincheng $\mathrm{Liu}^{\ddagger}$
}

October 28, 2013

\begin{abstract}
An asymptotic analysis of the tail probabilities for the dynamics of a soliton wave $U(x, t)$ under a stochastic time-dependent force is developed. The dynamics of the soliton wave $U(x, t)$ is described by the Korteweg-de Vries Equation with homogeneous Dirichlet boundary conditions under a stochastic time-dependent force, which is modeled as a time-dependent Gaussian noise with amplitude $\epsilon$. The tail probability we considered is $w(b):=P\left(\sup _{t \in[0, T]} U(x, t)>b\right)$, as $b \rightarrow \infty$, for some constant $T>0$ and a fixed $x$, which can be interpreted as tail probability of the amplitude of water wave on shallow surface of a fluid or long internal wave in a density-stratified ocean. Our goal is to characterize the asymptotic behaviors of $w(b)$ and to evaluate the tail probability of the event that the soliton wave exceeds a certain threshold value under a random force term. Such rare-event calculation of $w(b)$ is very useful for fast estimation of the risk of the potential damage that could caused by the water wave in a density-stratified ocean modeled by the stochastic KdV equation. In this work, the asymptotic approximation of the probability that the soliton wave exceeds a high-level $b$ is derived. In addition, we develop a provably efficient rare-event simulation algorithm to compute $w(b)$. The efficiency of the algorithm only requires mild conditions and therefore it is applicable to a general class of Gaussian processes and many diverse applications.
\end{abstract}

\section{Introduction}

It is well known that the Korteweg-de Vries $(\mathrm{KdV})$ equation can be used to model the evolution in time of long, unidirectional, weakly nonlinear water waves on shallow surface of a fluid, long internal waves in a density-stratified ocean, acoustic waves on a crystal lattice or ion accoustic wave phenomena in plasma dynamics. This paper focuses on the case when the forcing term of the $\mathrm{KdV}$ equation is random, which is a natural approach,

\footnotetext{
*xuxxx360@umn.edu, School of Statistics, University of Minnesota, Minneapolis, MN 55455, USA

†'guang.lin@pnnl.gov, Computational Sciences and Mathematics Division, Pacific Northwest National Laboratory, Richland, WA 99352 USA

${ }^{\ddagger}$ jcliu@stat.columbia.edu, Department of Statistics, Columbia University, New York, NY 10027 USA
} 
for instance, if we assume that the exterior pressure is generated by a turbulent velocity field. The stochastic KdV equation also can be employed to describe noisy plasmas, which has been studied mostly theoretically in the last two decades, see Wadati (1983); Herman (1990); Debussche and Printems (1999); Garnier (2001, 1993).

Understanding the probabilistic properties of the stochastic responses of stochastic $\mathrm{KdV}$ equation is one of the central objectives of this work. In plasma physics, for instance, physicists are interested in obtaining the averaged effects as well as the variation of the amplitude of ion-acoustic waves in noisy plasmas. In this paper, we are interested in rareevents associated with stochastic KdV equation. The analyses are crucial to the evaluation of the tail probabilities as well as the understanding of model response under extremeconditions. The problem motivating the current study is to investigate the tail probability of the event that the soliton wave exceeds a certain threshold value that tends to infinity under a stochastic time-dependent force. This probability qualitatively evaluates the risk of the potential damage that could caused by such soliton waves. This work provides a computationally viable way of evaluating the tail probabilities and a qualitative description of the behavior of the stochastic KdV equation given that the rare-event occurs.

It was Washimi and Taniuti (1966) who established rigorously that the KdV equation could be used to model the propagation of small-amplitude ion-acoustic waves. The stability properties of the solitons exhibited by certain nonlinear differential equations was first discovered by Zabusky and Kruskal (1965) in their classical study. The findings were confirmed later by the theory developed by Gardner and co-workers Gardner et al. (1967).

An exact solution for additive time-dependent white Gaussian noise was obtained by Wadati (1983). It was shown in Wadati (1983) that the mean single-soliton should behave as a Gaussian packet with width increasing as $t^{3 / 2}$ and amplitude decreasing as $t^{-3 / 2}$ at long-time. This work was later extended and exact multisoliton solutions was obtained by Wadati and Akutsu (1984). Wadati and Akutsu (1984) indicated that the mean soliton width increases only as $t^{1 / 2}$. Additionally, the theoretical results for the diffusion of solitons under the effect of multiplicative noise with long range correlation was obtained by Iizuka (1993). Later Iizuka's work Iizuka (1993) was confirmed by Scalerandi and Romano's numerical study (Scalerandi and Romano, 1998).

One important contribution to nonlinear random fields was made by the Inverse Scattering Technique (IST). It allows the construction of the exact solvable nonlinear stochastic equations. Various perturbation approaches have been developed based on the IST. Gardner et al. (1967) presented the first nonlinear evolution equation integrated by the inverse scattering scheme for KdV equation. Various foundations of IST can be found in Calogero and Degasperis (1982); Ablowitz and Segur (1981); Faddeev and Takhtajan (1987); Dodd et al. (1982); Newell (1985). Additionally, the books of Konotop and Vazquez (1994) and Novikov et al. (1980) study how IST is applied to the KdV equation. Furthermore, direct numerical simulation studies of the KdV equation and stochastic KdV are investigated in Debussche and Printems (1999); Lin et al. (2006). The primary objective of this work is to design efficient algorithms for fast rare-event calculations and employ such algorithms 
for rare-event calculation of stochastic $\mathrm{KdV}$ equation.

In this paper, we focus on investigating the one-dimensional KdV equation, with timedependent noise and homogeneous Dirichlet boundary conditions, in the form:

$$
\begin{aligned}
& u_{t}-6 u u_{x}+u_{x x x}=\varepsilon \xi(t), \quad x \in \mathbb{R} \\
& u(x, t=0)=-2 \kappa^{2} \operatorname{sech}^{2}(\kappa x),
\end{aligned}
$$

where $\kappa$ is the wave number, $\xi$ is the time-dependent Gaussian noise with mean zero, $\varepsilon$ is the amplitude, and $\operatorname{sech}(\cdot)$ is the hyperbolic secant function defined as $\operatorname{sech}(x)=2 /\left(e^{x}+e^{-x}\right)$. Write $f(t)=\varepsilon \int_{0}^{t} \xi(s) d s$. Note that $f$ is a mean zero Gaussian random process in $t$ with variance zero at $t=0$. The analytical solution to the $\mathrm{KdV}$ equation (1) is

$$
U(x, t)=f(t)-2 \kappa^{2} \operatorname{sech}^{2}\left\{\kappa\left(x-4 \kappa^{2} t\right)+6 \kappa \int_{0}^{t} f(s) d s\right\} .
$$

In this paper we are interested in the following tail probability

$$
w(b):=P\left(\sup _{t \in[0, T]} U(x, t)>b\right), \quad \text { as } b \rightarrow \infty,
$$

for a fixed $x$ and some constant $T>0$, which can be interpreted as the tail probability of the amplitude of water waves on shallow surface of a fluid or long internal waves in a density-stratified ocean. Our goal is to characterize the asymptotic behaviors of $w(b)$ and evaluate the tail probability of the event that the soliton wave $U(x, t)$ for a fixed $x$ and some constant $T>0$ exceeds a certain threshold value $b>0$ under a random force term. Such a rare-event calculation of $w(b)$ is very useful for fast estimation of the risk of the potential damage that could caused by the water wave in a density-stratified ocean modeled by the stochastic KdV equation. Given that the probability is always evaluated at a fixed location $x$, we omit the location index $x$ in $U(x, t)$ and write it as $U(t)$.

The solution function $U(t)$ takes the form of a non-linear functional of a Gaussian random field $f(t), t \in[0, T]$. Due to the non-linearity of $U(t)$, the tail asymptotic analysis of $w(b)$ are difficult and accurate approximations of $w(b)$ have not yet been developed in the literature. Therefore, rare-event simulation serves as an appealing alternative from the computational point of view in that the design and the analysis do not require very sharp approximations of $w(b)$. This paper, to the authors' best knowledge, is the first analysis of the extreme behavior of $U(t)$ for a general class of Gaussian random fields. The main contribution of this paper is to develop a provably efficient rare-event simulation algorithm to compute $w(b)$. The efficiency of the algorithm only requires that $f(t)$ is uniformly Hölder continuous on $[0, T]$. Therefore, the results are applicable to essentially all the Gaussian processes practically in use.

There is a rich rare-event simulation literature. An incomplete list of recent works includes Asmussen and Kroese (2006); Dupuis et al. (2007); Blanchet and Glynn (2008); Blanchet and Liu (2008); Blanchet et al. (2008). Recently, Adler et al. (2012) considered 
the supremum of general Hölder continuous Gaussian random fields and proposed efficient simulation algorithms based on the change of measure technique. Asymptotic tail behaviors of convex functionals of Gaussian random fields have also been studied in the literature; see Liu (2012); Liu and Xu (2012b, 2013b, 2012a, 2013a) for tail behaviors of exponential functionals of Gaussian random fields and the corresponding rare-event simulation analysis.

Central to our efficient simulation algorithm is the construction of a change of measure. Our proposed change of measure is not of a classical exponential-tilting form. Through this new measure, the proposed estimators basically reduce the rare-event calculations associated to $w(b)$ to calculations that are roughly comparable to the evaluation in which no rare-event is involved. To illustrate this, we perform the corresponding complexity analysis of the proposed estimators. This consists of two elements. Firstly, since $f$ considered in this paper is continuous, exact simulation of the entire field is usually impossible. Thus, we need to use a discrete object to approximate the continuous field and the bias caused by the discretization needs to be well controlled relative to $w(b)$. The second part of our analysis is the variance control, that is, to provide a bound of the second moments of the (discrete) importance sampling estimators.

The paper is organized as follows. In Section 1, we provide a general introduction. Efficient algorithms and theorems for rare-event simulation are introduced in Section 2. In Section 3, we present simulation results for the rare-event probabilities of stochastic KdV equation. A brief summary is given in Section 4. Proof of main results is provided in Section 5 .

\section{Main results}

\subsection{Rare-event Simulation}

Throughout this paper, we are interested in efficiently computing $w(b)$, which goes to 0 as $b \rightarrow \infty$. In the context of rare-event simulations, it is more meaningful to consider the computational error relative to $w(b)$. A well accepted efficiency concept is the so-called polynomial efficiency; see definition 2.1 in Adler et al. (2012).

Definition 1 A family of Monte Carlo estimators $\left\{L_{b}: b \geq b_{0}\right\}$ is said to be polynomially efficient in estimating $w(b)$ if $E L_{b}=w(b)$, and there exists a constant $q>0$ such that

$$
\sup _{b \geq b_{0}} \frac{\operatorname{Var}\left(L_{b}\right)}{w^{2}(b)|\log w(b)|^{q}}<\infty
$$

Suppose that a polynomially efficient estimator $L_{b}$ has been obtained. Let $\left\{L_{b}^{(j)}: j=\right.$ $1, \ldots, n\}$ be i.i.d. copies of $L_{b}$ and $Z_{b}=\frac{1}{n} \sum_{j=1}^{n} L_{b}^{(j)}$ be the averaged estimator that has a relative mean squared error $\operatorname{Var}^{1 / 2}\left(L_{b}\right) / n^{1 / 2} w(b)$. A simple consequence of Chebyshev's 
inequality yields that for any $\eta>0$

$$
P\left(\left|Z_{b} / w(b)-1\right| \geq \eta\right) \leq \frac{\operatorname{Var}\left(L_{b}\right)}{n \eta^{2} w^{2}(b)} .
$$

The limit (3) suggests that $\operatorname{Var}\left(L_{b}\right)=O\left(w^{2}(b)|\log w(b)|^{q}\right)$. Therefore for any positive $\eta$ and $\delta$, in order to achieve the following relative accuracy

$$
P\left(\left|Z_{b} / w(b)-1\right|>\eta\right)<\delta,
$$

it is sufficient to generate $n=O\left(\eta^{-2} \delta^{-1}|\log w(b)|^{q}\right)$ i.i.d. copies of $L_{b}$. In Proposition 10, we will show that $|\log w(b)|=O\left(b^{2}\right)$. Therefore, a polynomially efficient estimator for $w(b)$ only requires to generate $n=O\left(\eta^{-2} \delta^{-1} b^{2 q}\right)$ i.i.d. copies. Compared with the crude Monte Carlo simulation, which requires $n=O\left(\eta^{-2} \delta^{-1} w(b)^{-1}\right)$, polynomially efficient estimators are exponentially faster and therefore substantially reduce the computational cost.

To construct such polynomially efficient estimators, in this paper, we use importance sampling for the variance reduction; see Chapter V in Asmussen and Glynn (2007). It is based on the following identity

$$
w(b)=E\left(1_{\left(\sup _{t} U(t)>b\right)}\right)=E^{Q}\left(1_{\left(\sup _{t} U(t)>b\right)} \frac{d P}{d Q}\right),
$$

where $1_{(\cdot)}$ is the indicator function, $Q$ is a probability measure such that $d P / d Q$ is well defined (finite) on the set $\left\{\sup _{t} U(t)>b\right\}$, and we use $E$ and $E^{Q}$ to denote the expectations under the measures $P$ and $Q$ respectively. Then, the random variable

$$
L_{b}=1_{\left(\sup _{t} U(t)>b\right)} \frac{d P}{d Q}
$$

is an unbiased estimator of $w(b)$ under the measure $Q$.

Consider the conditional distribution $\mathcal{Q}(\cdot)=P\left(\cdot \mid \sup _{t} U(t)>b\right)$. The corresponding likelihood ratio $d P / d \mathcal{Q} \equiv w(b)$ on the set $\left\{\sup _{t} U(t)>b\right\}$ has zero variance under $\mathcal{Q}$. Thus, $\mathcal{Q}$ is also called the zero-variance change of measure. Note that this change of measure is of no practical value in that its implementation requires the computation of the probabilities $w(b)$. Nonetheless, the measure $\mathcal{Q}$ provides a guideline for the construction of an efficient change of measure to compute the probabilities $w(b)$. Therefore, the task lies in constructing a change of measure $Q$ that is a good approximation of $\mathcal{Q}$. In addition, from the computational point of view, we should also be able to numerically compute $L_{b}$ and to simulate $f$ from $Q$.

Besides the variance control, another important issue is the bias control. The random fields considered in this paper are continuous processes. Direct simulation is usually not feasible. Therefore, we need to set up an appropriate discretization scheme to approximate the continuous objects. The bias caused by the discretization also needs to be controlled 
relative to $w(b)$. Suppose that a biased estimator $\tilde{L}_{b}$ has been constructed for $w(b)$ such that $E\left(\tilde{L}_{b}\right)=\tilde{w}(b)$. Thus, the computation error can be decomposed as follows

$$
\left|\frac{\tilde{L}_{b}}{w(b)}-1\right| \leq \frac{\left|\tilde{L}_{b}-\tilde{w}(b)\right|}{w(b)}+\frac{|\tilde{w}(b)-w(b)|}{w(b)} .
$$

The first term on the right-hand-side is controlled by the relative variance of $\tilde{L}_{b}$ and the second term is the bias relative to $w(b)$. Both the bias and the variance control will be carefully analyzed in the following sections relative to $w(b)$. The overall computational complexity is then the necessary number of i.i.d. replicates for $\tilde{L}_{b}$ multiplied by the computational cost to generate one $\tilde{L}_{b}$.

\subsection{Change of Measure and Simulation Algorithm}

For the implementation, we introduce a suitable discretization scheme on $[0, T]$. Let

$$
T_{N}=\left\{t_{1}, \cdots, t_{N}\right\} \text { with } t_{i}=\frac{i}{N} T, \quad i=1, \cdots, N .
$$

We use

$$
w_{N}(b)=P\left(\sup _{i=1, \cdots, N} U\left(t_{i}\right)>b\right)
$$

as an approximation of $w(b)$. We will show in Section 2.3 that the bias caused by the discretization is well controlled relative to $w(b)$ with properly chosen discretization size $N$. In the following, we introduce an efficient simulation algorithm to estimate $w_{N}(b)$.

We estimate $w_{N}(b)$ by importance sampling, which is based on the following change of measure $Q_{N}$. We characterize the measure $Q_{N}$ in two ways. First, we describe the simulation of the discretized process $f\left(t_{i}\right), i=1, \cdots, N$, from $Q_{N}$ following a three-step procedure.

Step 1 Sample a point $\tau_{b} \in\{1, \cdots, N\}$ according to the following probability measure

$$
P\left(\tau_{b}=i\right)=\frac{P\left(f\left(t_{i}\right)>b\right)}{\sum_{j=1}^{N} P\left(f\left(t_{j}\right)>b\right)} .
$$

Step 2 With a generated $\tau_{b}$, we sample $f\left(\tau_{b}\right)$ conditional on $f\left(\tau_{b}\right)>b$.

Step 3 We proceed to sample $f\left(t_{j}\right), t_{j} \neq \tau_{b}$, from the original conditional distribution under $P$ given $\tau_{b}$ and $f\left(\tau_{b}\right)$.

The above simulation description induces the measure $Q_{N}$, taking the form

$$
Q_{N}(\cdot)=\sum_{\tau_{b}=i}^{N} P\left(\cdot \mid f\left(t_{i}\right)>b\right) P\left(\tau_{b}=i\right) .
$$


Then the corresponding Radon-Nikodym derivative between $Q_{N}$ and $\mathrm{P}$ is

$$
\frac{d Q_{N}}{d P}=\frac{\sum_{i=1}^{N} 1_{\left(f\left(t_{i}\right)>b\right)}}{\sum_{i=1}^{N} P\left(f\left(t_{i}\right)>b\right)},
$$

which gives the second characterization of the change of measure.

The measure $Q_{N}$ is constructed such that the behavior of $f$ under $Q_{N}$ mimics the tail behavior of $f$ given the rare event $\left\{\sup _{i} U\left(t_{i}\right)>b\right\}$, a good approximation of $\left\{\sup _{t} U(t)>\right.$ $b$, under $P$. According to the above simulation procedure, a random variable $\tau_{b}$ is first generated, then $f\left(\tau_{b}\right)$ is simulated at level $O(b)$. Under the zero-variance change of measure, the large value of the supremum $U(t)$ is mostly caused by the high excursion of $f(t)$ at one location. The random index $\tau_{b}$ is adopted to search the maximum of $f(t)$ over the interval $[0, T]$. It worth emphasizing that $\tau$ is not necessarily the exact maximum but should be very close to it.

Based on the above discussion, we have the importance sampling estimator taking the form (see (6))

$$
\tilde{L}_{b}=\frac{d P}{d Q_{N}} 1_{\left(\sup _{i} U\left(t_{i}\right)>b\right)}=\frac{\sum_{i=1}^{N} P\left(f\left(t_{i}\right)>b\right)}{\sum_{i=1}^{N} 1_{\left(f\left(t_{i}\right)>b\right)}} 1_{\left(\sup _{i} U\left(t_{i}\right)>b\right)} .
$$

and its second moment (under the measure $Q_{N}$ ) equals

$$
E^{Q_{N}}\left[\tilde{L}_{b}^{2}\right]=E^{Q_{N}}\left[\left(\frac{\sum_{i=1}^{N} P\left(f\left(t_{i}\right)>b\right)}{\sum_{i=1}^{N} 1_{\left(f\left(t_{i}\right)>b\right)}}\right)^{2} ; \sup _{i} U\left(t_{i}\right)>b\right] .
$$

Note that under $Q_{N}$ we always have $\max _{i} f\left(t_{i}\right)>b$ and therefore the denominator of $\tilde{L}_{b}$ is at least 1 and well defined.

The corresponding simulation algorithm is given as follows.

Algorithm 2 The algorithm has two steps:

Step 1: Simulate $\tau_{b}$ from $\{1, \ldots, N\}$ according to the distribution function (7) and generate $f\left(t_{\tau_{b}}\right)$ conditional on $f\left(t_{\tau_{b}}\right)>b$. Given $\left(\tau_{b}, f\left(t_{\tau_{b}}\right)\right)$, simulate $\left(f\left(t_{1}\right), \cdots, f\left(t_{\tau_{b}-1}\right)\right.$, $\left.f\left(t_{\tau_{b}+1}\right), \cdots, f\left(t_{N}\right)\right)$ from the original conditional distribution under the measure $P$.

Step 2: Compute and output

$$
\tilde{L}_{b}=\frac{\sum_{i=1}^{N} P\left(f\left(t_{i}\right)>b\right)}{\sum_{i=1}^{N} 1_{\left(f\left(t_{i}\right)>b\right)}} 1_{\left(\sup _{i} U\left(t_{i}\right)>b\right)} .
$$

We can see that $\tilde{L}_{b}$ is an unbiased estimator of $w_{N}(b)$. The computation of the above estimator and the event $\sup _{i} U\left(t_{i}\right)>b$ only consists of $\left\{f\left(t_{i}\right): i=1, \ldots, N\right\}$ and therefore is easy to perform. We present the corresponding efficiency results in the next section. 


\subsection{Efficiency Results}

We first state the following technical conditions required for our main results.

A1 $f(t)$ is almost surely continuous with respect to $t$ and admits $E[f(t)]=0$ and $E\left[f^{2}(t)\right]=\sigma^{2}(t)$ for all $t \in T$; in addition, we assume that $\sigma(0)=0$;

A2 There exist $\delta, \kappa_{H}>0$, and $\beta \in(0,1]$ such that, for all $|s-t|<\delta$, the variance functions satisfy

$$
|\sigma(t)-\sigma(s)| \leq \kappa_{H}|s-t|^{\beta}
$$

A3 For each $s, t \in T$, let covariance function $C(s, t)=\operatorname{Cov}(f(s), f(t))>0$. For all $\left|s-s^{\prime}\right|<\delta$ and $\left|t-t^{\prime}\right|<\delta$, the covariance function satisfies

$$
\left|C(t, s)-C\left(t^{\prime}, s^{\prime}\right)\right| \leq \kappa_{H}\left(\left|t-t^{\prime}\right|^{2 \beta}+\left|s-s^{\prime}\right|^{2 \beta}\right) .
$$

Condition A1 assumes that $f$ has zero mean and variance function $\sigma^{2}(t)$. In fact, for Gaussian random fields with non-constant mean functions, our efficiency results are still expected to be applicable. The assumption that $\sigma(0)=0$ follows from the definition of $f$ as in the introduction. Conditions A2 and A3 essentially ensure that the process $f(t)$ is uniformly Hölder continuous, that is, for $|s-t|<\delta$, $\operatorname{Var}(f(s)-f(t)) \leq 2 \kappa_{H}|s-t|^{2 \beta}$. These assumptions are weak enough such that they accommodate essentially all Gaussian processes practically in use (fractional Brownian motion, smooth Gaussian processes, etc). Therefore, the algorithm developed in this paper is suitable for a wide range of applications.

As introduced in the previous section, we use $w_{N}(b)=P\left(\sup _{i=1, \cdots, N} U\left(t_{i}\right)>b\right)$ as an approximation of $w(b)$. The next theorem controls the bias.

Theorem 3 For any $\epsilon>0$ and $\eta>0$, there exist constants $\kappa_{0}, \kappa_{1}$ such that for all $b>\kappa_{1}|\log \eta|$ and $N=\kappa_{0}^{1 / \beta}|\log \eta|^{1 /(2 \beta)} \eta^{-1 / \beta} b^{(2+2 \epsilon) / \beta}$, we have

$$
\left|w_{N}(b)-w(b)\right|<\eta w(b) .
$$

Thanks to the continuity of $f(t)$, as $N$ tends to infinity, the lattice $T_{N}$ becomes dense in $[0, T]$ and therefore $w_{N}(b)$ gives a good approximation of $w(b)$ as shown in the above theorem. In particular, it provides a lower bound of $N$ such that $w_{N}(b) / w(b)$ is close enough to 1 and the relative bias can be controlled.

With $N$ chosen as in Theorem 3, we estimate $w_{N}(b)$ by importance sampling estimator $\tilde{L}_{b}$, as introduced in Algorithm 2. The next theorem controls the variance of the estimator $\tilde{L}_{b}$ and gives the polynomial efficiency result.

Theorem 4 Suppose that $N$ is chosen as in Theorem 3. There exists a finite $c_{0}$ such that

$$
E^{Q_{N}}\left[\tilde{L}_{b}^{2}\right] \leq c_{0} N^{2} w^{2}(b) .
$$


The above results show that the estimator $\tilde{L}_{b}$ in Algorithm 2 is polynomially efficient in estimating $w_{N}(b)$. To estimate $w(b)$, we simulate $n$ i.i.d. copies of $\tilde{L}_{b},\left\{\tilde{L}_{b}^{(j)}: j=1, \ldots, n\right\}$ and the final estimator is $Z_{b}=\frac{1}{n} \sum_{j=1}^{n} \tilde{L}_{b}^{(j)}$. The estimation error is

$$
\left|Z_{b}-w(b)\right| \leq\left|w_{N}(b)-w(b)\right|+\left|Z_{b}-w_{N}(b)\right| .
$$

The first term is controlled by Theorem 3, i.e., $\left|w_{N}(b)-w(b)\right| \leq \eta w(b)$ if we choose the discretization size

$$
N=O\left(|\log \eta|^{1 /(2 \beta)} \eta^{-1 / \beta} b^{2(1+\epsilon) / \beta}\right) .
$$

The second term of (10) is controlled by the discussion as in (4) if we choose the number of replicates

$$
n=O\left(\eta^{-2} \delta^{-1} N^{2}\right)
$$

The simulation of $\tilde{L}_{b}$ consists of generating a random vector of dimension $N$. Note that the complexity of computing the eigenvalues and eigenvectors of an $N$-dimension matrix is $O\left(N^{3}\right)$. Thus, the total computational complexity to achieve the prescribed accuracy in (5) is $O\left(N^{5} \eta^{-2} \delta^{-1}\right)$.

\section{Simulation}

We use simulation results to illustrate the performance of the proposed algorithm. We assume that $f$ is a Brownian motion on $[0,1]$ and we take $T_{N}=\{i / 100: i=1, \cdots, 100\}$ with discretization size $N=100$. The detailed simulation is described in the next steps.

1. Generate a random variable $\iota$ from $\{1,2, \cdots, 100\}$.

2. Simulate $f\left(\frac{\iota}{100}\right)$ conditional on $f\left(\frac{\iota}{100}\right)>b$.

3. Given $\iota$ and $f\left(\frac{\iota}{100}\right)$, simulate $\left\{f\left(\frac{i}{100}\right), i=1, \cdots, \iota-1, \iota+1, \cdots, 100\right\}$.

The estimated tail probabilities $w(b)$ along with the estimated standard deviations $\operatorname{St} d^{Q}\left(\tilde{L}_{b}\right)$ are shown in Table 1 . All the results are based on $10^{3}$ independent simulations. The standard deviation of the final estimate (in the column "Est.") is the reported standard deviation (in the column of "Std.") divided by $\sqrt{1000}$ ( in the column "Std $/ \sqrt{1000 ") . ~}$

The simulation results show that the coefficient of variation $\left(\operatorname{Std}^{Q}\left(L_{b}\right) / w(b)\right)$ increases as the tail probability becomes smaller. Nevertheless, the coefficient of variation stays reasonably small when the probability is as small as $10^{-13}$. To validate the simulation results, we use crude Monte Carlo for $b=3$. Based on $10^{5}$ independent simulations, the estimated tail probability is $6.4 \mathrm{e}-4$ (Std. 8.0e-05), which is consistent with that computed by the importance sampling estimator. We also use discretization size $N=500$ to check the simulation bias introduced by the discretization scheme. The simulation results are given in Table 2. Again these results are consistent with the simulation results in Table 1. 


\begin{tabular}{|c|c|c|c|c|}
\hline$b$ & Est. & Std. & Std./Est. & Std. $/ \sqrt{1000 .}$ \\
\hline$b=3$ & $5.51 \mathrm{e}-04$ & $6.10 \mathrm{e}-04$ & 1.11 & $1.93 \mathrm{e}-05$ \\
$b=5$ & $1.11 \mathrm{e}-07$ & $1.42 \mathrm{e}-07$ & 1.28 & $4.49 \mathrm{e}-09$ \\
$b=7$ & $4.10 \mathrm{e}-13$ & $6.07 \mathrm{e}-13$ & 1.48 & $1.92 \mathrm{e}-14$ \\
\hline
\end{tabular}

Table 1: Estimates of $w(b)$ on $[0,1]$. All results are based on $10^{3}$ independent simulations and discretization size $N=100$.

\begin{tabular}{|c|c|c|c|}
\hline$b$ & Est. & Std. & Std./Est. \\
\hline$b=3$ & $5.35 \mathrm{e}-04$ & $5.73 \mathrm{e}-04$ & 1.07 \\
$b=5$ & $1.11 \mathrm{e}-07$ & $1.58 \mathrm{e}-07$ & 1.43 \\
$b=7$ & $4.08 \mathrm{e}-13$ & $8.08 \mathrm{e}-13$ & 1.98 \\
\hline
\end{tabular}

Table 2: Estimates of $w(b)$ on $[0,1]$. All results are based on $10^{3}$ independent simulations and discretization size $N=500$.

\section{Summary}

We developed a provably efficient asymptotic rare-event simulation algorithms to compute the tail probabilities of the amplitude of water waves on shallow surface of a fluid or long internal waves in a density-stratified ocean under a stochastic time-dependent force. The dynamics of the soliton wave is described by the KdV equation with homogeneous Dirichlet boundary conditions under a stochastic time-dependent force, which is modeled as a time-dependent Gaussian noise with amplitude $\epsilon$. We develop a provably efficient rare-event simulation algorithm of the tail probabilities that the soliton wave exceeds a high-level $b$ that tends to infinity. The simulation results show that the coefficient of variation $\left(S t d^{Q}\left(L_{b}\right) / w(b)\right)$ increases as the tail probability becomes smaller. Nevertheless, the coefficient of variation stays reasonably small when the probability is as small as $10^{-13}$. Additionally, we also provide the proofs of all the supporting lemmas and theorems for the bounds of the tail probability $w(b)$. Such rare-event calculation of $w(b)$ is very useful for fast estimation of the risk of the potential damage that could caused by the water wave in a density-stratified ocean modeled by the stochastic KdV equation. The efficiency of the algorithm only requires mild conditions and therefore it is applicable to a general class of Gaussian processes and many diverse applications. 


\section{$5 \quad$ Proof of Main Results}

\subsection{Proofs of main theorems}

Proof of Theorem 3. For any $\eta, \epsilon>0$ and a large constant $\kappa_{0}>0$ (to be determined later), let

$$
\mathcal{L}=\left\{f(\cdot) \in \mathcal{C}(T): \sup _{s, t:|t-s| \leq N^{-1}}|f(s)-f(t)| \leq \eta \kappa_{0}^{-1 / 2} b^{-(1+\epsilon)}\right\}
$$

where $\mathcal{C}(T)$ is the set of all continuous functions on $[0, T]$ and $N$ is chosen as in the statement of the theorem. The following lemma suggests that for our analysis we only need to focus on the set $\mathcal{L}$.

Lemma 5 Under the conditions in Theorem 3, for any $\epsilon, \eta>0$, there exists a constant $\kappa_{0}$ such that, for $N=\kappa_{0}^{1 / \beta}|\log \eta|^{1 /(2 \beta)} \eta^{-1 / \beta} b^{(2+2 \epsilon) / \beta}$, we have

$$
P\left(\sup _{t \in[0, T]} U(t)>b, \mathcal{L}^{c}\right) \leq \eta w(b) .
$$

Note that $\max _{i} U\left(t_{i}\right) \leq \sup _{t} U(t)$. We have the following upper bound for the bias of $w_{N}(b)$ :

$$
\begin{aligned}
\left|w_{N}(b)-w(b)\right| & \leq\left|P\left(\max _{i} U\left(t_{i}\right)>b, \mathcal{L}\right)-P\left(\sup _{t} U(t)>b, \mathcal{L}\right)\right|+2 \eta w(b) \\
& =P\left(\max _{i} U\left(t_{i}\right)<b, \sup _{t} U(t)>b, \mathcal{L}\right)+2 \eta w(b) .
\end{aligned}
$$

Applying the Borel-TIS lemma (Lemma 8), we have that there is a constant $M:=$ $|\log \eta|^{1 / 2} \kappa_{0}^{1 / 3}$ such that for $\kappa_{0}$ large enough

$$
P\left(\sup _{t} f(t)>M b\right) \leq \exp \left(-\frac{\left(M b-E\left[\sup _{t} f(t)\right]\right)^{2}}{2 \sigma_{T}^{2}}\right)<\eta w(b),
$$

where $\sigma_{T}=\sup _{t \in[0, T]} \sigma(t)$ and the second inequality follows from Proposition 10 (stated on page 14), which states that $b^{-2} \log w(b) \rightarrow-1 /\left(2 \sigma_{T}^{2}\right)$. Therefore, we have that

$$
\left|w_{N}(b)-w(b)\right| \leq P\left(\max _{i} U\left(t_{i}\right)<b<\sup _{t} U(t), \sup _{t} f(t)<M b, \mathcal{L}\right)+3 \eta w(b) .
$$

Then on the set $\mathcal{L} \cap\left\{\sup _{t} f(t)<M b\right\}$, we can show

$$
\left|\max _{i} U\left(t_{i}\right)-\sup _{t} U(t)\right| \leq \sup _{t:\left|t-t_{i}\right| \leq N^{-1}}\left|f\left(t_{i}\right)-f(t)\right|+O(1) N^{-1} M b \leq 2 \eta \kappa_{0}^{-1 / 2} b^{-(1+\epsilon)},
$$


where $O(1)$ term is a bounded constant, and the last inequality holds for large $\kappa_{0}$. Therefore we obtain the following upper bound

$$
\left|w_{N}(b)-w(b)\right| \leq P\left(b-2 \eta \kappa_{0}^{-1 / 2} b^{-(1+\epsilon)}<\sup _{t} U(t)<b+2 \eta \kappa_{0}^{-1 / 2} b^{-(1+\epsilon)}, \mathcal{L}\right)+3 \eta w(b) .
$$

Let $c_{b}=2 \eta \kappa_{0}^{-1 / 2} b^{-(1+\epsilon)}$. We have the following lemma to bound the probability term in the above display, whose proof is provided in Section 5.2.

Lemma 6 Under the conditions of Theorem 3, we have

$$
w(b)=(1+o(1)) P\left(\sup _{t \in[0, T]} f(t)>b\right) .
$$

In addition there exists a constant $c$ such that

$$
P\left(b-c_{b}<\sup _{t \in[0, T]} U(t)<b+c_{b}, \mathcal{L}\right) \leq c c_{b} F^{\prime}\left(b-c_{b}\right),
$$

where $F^{\prime}(x)$ is the probability density function of $\sup _{t \in[0, T]} f(t)$.

The following lemma provides an upper bound of the density function $F^{\prime}(x)$, whose proof follows from Ehrhard Inequality (Ehrhard, 1983); see also Chapter 4 in Bogachev (1998).

Lemma 7 Under the conditions of Theorem 3, let $F^{\prime}(x)$ be the probability density function of $\sup _{t \in[0, T]} f(t)$. Then $F^{\prime}(x)$ exists almost everywhere. Moreover, as $x$ goes to infinity,

$$
F^{\prime}(x)=(1+o(1)) \sigma_{T}^{-1} x w(x) .
$$

Lemma 7 and (15) imply that there exists a constant $c$ such that

$$
\left|w_{N}(b)-w(b)\right| \leq c \eta \kappa_{0}^{-1 / 2} b^{-\epsilon} w\left(b-c_{b}\right)+3 \eta w(b) .
$$

By (14), we have the following approximation

$$
w\left(b-c_{b}\right)=(1+o(1)) P\left(\sup _{t} f(t)>b-c_{b}\right) .
$$

Apply Lemma 7 again and we have

$$
P\left(\sup _{t} f(t)>b-c_{b}\right)=(1+o(1)) P\left(\sup _{t} f(t)>b\right)=(1+o(1)) w(b) .
$$


Therefore $w\left(b-c_{b}\right)=(1+o(1)) w(b)$ and for $\kappa_{0}$ large enough, $\left|w_{N}(b)-w(b)\right| \leq 4 \eta w(b)$. Then, we can redefine $\eta$ and the constant $\kappa_{0}$ and obtain the conclusion.

Proof of Theorem 4. We have

$$
\begin{aligned}
E^{Q_{N}}\left[\tilde{L}_{b}^{2}\right] & =E^{Q_{N}}\left[\left(\frac{\sum_{i=1}^{N} P\left(f\left(t_{i}\right)>b\right)}{\sum_{i=1}^{N} 1_{\left(f\left(t_{i}\right)>b\right)}}\right)^{2} ; \sup _{i} U\left(t_{i}\right)>b\right] \\
& \leq\left(\sum_{i=1}^{N} P\left(f\left(t_{i}\right)>b\right)\right)^{2} \\
& \leq N^{2} \max _{i} P\left(f\left(t_{i}\right)>b\right)^{2} \\
& \leq N^{2} P\left(\max _{i} f\left(t_{i}\right)>b\right)^{2} \leq N^{2} P\left(\sup _{t \in[0, T]} f(t)>b\right)^{2}
\end{aligned}
$$

Lemma 6 shows that $w(b)=(1+o(1)) P\left(\sup _{t} f(t)>b\right)$. Thus, there exists some constant $c_{0}>0$ such that

$$
E^{Q_{N}}\left[\tilde{L}_{b}^{2}\right] \leq c_{0} N^{2} w(b)^{2}
$$

which gives the desired result.

\subsection{Proof of Lemmas}

In this section, we present the proofs of all the supporting lemmas in the main proof. We start with some useful results. The following lemma is known as the Borel-TIS lemma, which is proved independently by Borell (1975) and Tsirelson, Ibragimov and Sudakov (1976).

Lemma 8 (Borel-TIS) Let $f(t), t \in \mathcal{U}, \mathcal{U}$ is a parameter set, be a mean zero Gaussian random field. $f$ is almost surely bounded on $\mathcal{U}$. Then, $E\left[\sup _{\mathcal{U}} f(t)\right]<\infty$, and

$$
P\left(\sup _{t \in \mathcal{U}} f(t)-E\left[\sup _{t \in \mathcal{U}} f(t)\right] \geq b\right) \leq \exp \left(-\frac{b^{2}}{2 \sigma_{\mathcal{U}}^{2}}\right)
$$

where $\sigma_{\mathcal{U}}^{2}=\sup _{t \in \mathcal{U}} \operatorname{Var}[f(t)]$.

The Borel-TIS lemma provides a very general bound of the tail probability. In most cases, $E\left[\sup _{t} f(t)\right]$ is much smaller than $b$. Thus, for $b$ sufficiently large, the tail probability can be further bounded by

$$
P\left(\sup _{t} f(t)>b\right) \leq \exp \left(-\frac{b^{2}}{4 \sigma_{T}^{2}}\right) .
$$

In particular, the following result by Dudley (1973) (c.f. Theorem 6.7 in Adler, Blanchet and Liu, 2012) is often used to control $E\left[\sup _{t \in \mathcal{U}} f(t)\right]$. 
Lemma 9 Let $\mathcal{U}$ be a compact subset of $R^{d}$, and let $\{f(t) t \in \mathcal{U}\}$ be a mean zero, continuous Gaussian random field. Define the canonical metric $d$ on $\mathcal{U}$ as

$$
d(s, t)=\sqrt{E[f(t)-f(s)]^{2}}
$$

and put $\operatorname{diam}(\mathcal{U})=\sup _{s, t \in \mathcal{U}} d(s, t)$, which is assumed to be finite. Then there exists a finite universal constant $\kappa>0$ such that

$$
E\left[\max _{t \in \mathcal{U}} f(t)\right] \leq \kappa \int_{0}^{\operatorname{diam}(\mathcal{U}) / 2}[\log (\mathcal{N}(\varepsilon))]^{1 / 2} d \varepsilon
$$

where the entropy $\mathcal{N}(\varepsilon)$ is the smallest number of d-balls of radius $\varepsilon$ whose union covers $\mathcal{U}$.

We can easily obtain the following bounds for the tail probability $w(b)$. The first result is due to the fact that $f(t)-2 \kappa^{2} \leq U(t) \leq f(t)$ and the second result follows from a direct application of the Borel-TIS lemma.

Proposition 10 Let $\sigma_{T}=\sup _{t \in[0, T]} \sigma(t)$. Then,

$$
P\left(\sup _{t \in[0, T]} f(t)>b+2 \kappa^{2}\right) \leq w(b) \leq P\left(\sup _{t \in[0, T]} f(t)>b\right),
$$

and as $b \rightarrow \infty$,

$$
b^{-2} \log w(b) \rightarrow-\frac{1}{2 \sigma_{T}^{2}}
$$

We proceed to prove the supporting lemmas in the previous section.

Proof of Lemma 5. Note that

$$
P\left(\sup _{t} U(t)>b, \mathcal{L}^{c}\right) \leq P\left(\sup _{t} f(t)>b, \mathcal{L}^{c}\right)
$$

therefore we only need to show $P\left(\sup _{t} f(t)>b, \mathcal{L}^{c}\right) \leq \eta w(b)$.

On set $\mathcal{L}^{c}, \sup _{s, t:|t-s| \leq N^{-1}}|f(s)-f(t)|>\eta \kappa_{0}^{-1 / 2} b^{-(1+\epsilon)}$. For random variable $f(s)-f(t)$, the variance function has an upper bound:

$$
\begin{aligned}
\operatorname{Var}(f(s)-f(t))=2(1-C(s, t)) & \leq 2 \kappa_{H}|s-t|^{2 \beta} \\
& \leq 2 \kappa_{H} N^{-2 \beta}=2 \kappa_{H} \kappa_{0}^{-2}|\log \eta|^{-1} \eta^{2} b^{-4(1+\epsilon)}
\end{aligned}
$$


Then, for $\kappa_{0}$ big enough, the Borel-TIS lemma (Lemma 8) and Lemma 9 imply that there exists $\lambda>0$ such that

$$
\begin{aligned}
& P\left(\sup _{t} f(t)>b, \mathcal{L}^{c}\right) \\
\leq & P\left(\sup _{s, t:|t-s| \leq N^{-1}}|f(s)-f(t)|>\eta \kappa_{0}^{-1 / 2} b^{-(1+\epsilon)}\right) \\
\leq & 2 \exp \left(-\frac{\left(\eta \kappa_{0}^{-1 / 2} b^{-(1+\epsilon)}-E\left[\sup _{s, t:|t-s| \leq N^{-1}}(f(s)-f(t))\right]\right)^{2}}{4 \sigma_{T}^{2} \kappa_{H} \kappa_{0}^{-2}|\log \eta|^{-1} \eta^{2} b^{-4(1+\epsilon)}}\right) \\
\leq & 2 \exp \left(-\lambda|\log \eta| \kappa_{0} b^{2(1+\epsilon)}\right) .
\end{aligned}
$$

Therefore by the result that $b^{-2} \log w(b) \rightarrow-1 /\left(2 \sigma_{T}^{2}\right)$ from Proposition 10, we have $P\left(\sup _{t} f(t)>b, \mathcal{L}^{c}\right) \leq \eta w(b)$ when $\kappa_{0}$ is large.

Proof of Lemma 6. (i). For the first approximation result, thanks to Lemma 5, we only need to show that

$$
P\left(\sup _{t} U(t)>b, \mathcal{L}\right)=(1+o(1)) P\left(\sup _{t} f(t)>b\right) .
$$

Under conditions A1 and A2, there exists $\delta_{0}>0$ such that

$$
\sup _{0 \leq t \leq \delta_{0}} \sigma(t)<\sup _{0 \leq t \leq T} \sigma^{2}(t)=\sigma_{T}
$$

For any $\delta>0$, we have the following decomposition

$$
\begin{aligned}
& P\left(\sup _{t} U(t)>b, \mathcal{L}\right) \\
= & P\left(\sup _{t} U(t)>b, \sup _{t} f(t)>b, \mathcal{L}\right) \\
= & P\left(\sup _{t} U(t)>b, \inf _{\delta_{0}<t \leq T} \int_{0}^{t} f(s) d s<\delta b, \sup _{t} f(t)>b, \mathcal{L}\right) \\
& +P\left(\sup _{t} U(t)>b, \inf _{\delta_{0}<t \leq T} \int_{0}^{t} f(s) d s>\delta b, \sup _{t} f(t)>b, \mathcal{L}\right) .
\end{aligned}
$$

We consider the two terms in (18) one by one. Consider the following change of measure

$$
\frac{d \bar{Q}}{d P}=\int_{T} \frac{1}{T} \frac{e^{-(f(t)-b)^{2} /\left(2 \sigma^{2}(t)\right)}}{e^{-f(t)^{2} /\left(2 \sigma^{2}(t)\right)}} d t=\frac{1}{T} \int_{T} e^{b f(t) / \sigma^{2}(t)-b^{2} /\left(2 \sigma^{2}(t)\right)} d t .
$$


Under the measure $\bar{Q}, f$ can be considered to be generated in the following way: 1) Simulate a random index $\iota$ uniformly over $[0, T]$ with respect to the Lebesgue measure. 2) Given the realized $\iota$, simulate $f(\iota) \sim N\left(b, \sigma^{2}(\iota)\right)$. 3) Simulate the rest of the field $\{f(t): t \neq \iota\}$ from the original conditional distribution under $P$ given $(\iota, f(\iota))$. Note that under the measure $\bar{Q}$, conditional on $\iota$, we can write

$$
f(t)=C(t, \iota) b+g(t)
$$

where $g(t), t \in[0, T]$, is a Gaussian process such that it has the same distribution under the measure $\bar{Q}$ as that of $f$ under the measure $P$.

Use the above change of measure, we have

$$
\begin{aligned}
& P\left(\sup _{t} U(t)>b, \inf _{\delta_{0}<t \leq T} \int_{0}^{t} f(s) d s<\delta b, \sup _{t} f(t)>b, \mathcal{L}\right) \\
\leq & P\left(\inf _{\delta_{0}<t \leq T} \int_{0}^{t} f(s) d s<\delta b, \sup _{t} f(t)>b, \mathcal{L}\right) \\
= & E^{\bar{Q}}\left[\frac{d P}{d \bar{Q}} ; \sup _{t} f(t)>b, \inf _{\delta_{0}<t \leq T} \int_{0}^{t} f(s) d s<\delta b, \mathcal{L}\right] \\
= & E^{\bar{Q}}\left[\frac{1}{\int_{T} \frac{1}{T} e^{b f(t) / \sigma^{2}(t)-b^{2} /\left(2 \sigma^{2}(t)\right)} d t} ; \sup _{t} f(t)>b, \inf _{\delta_{0}<t \leq T} \int_{0}^{t} f(s) d s<\delta b, \mathcal{L}\right] .
\end{aligned}
$$

Therefore, there exists $\lambda>0$ such that on the set $\mathcal{L}$

$$
\begin{aligned}
& P\left(\int_{0}^{T} f(t) d t<\delta b, \sup _{t} f(t)>b, \mathcal{L}\right) \\
\leq & E^{\bar{Q}}\left[\frac{1}{\lambda N^{-1} e^{b \sup _{t} f(t) / \sigma_{T}^{2}-b^{2} /\left(2 \sigma_{T}^{2}\right)}} ; \inf _{\delta_{0}<t \leq T} \int_{0}^{t} f(s) d s<\delta b, \sup _{t} f(t)>b, \mathcal{L}\right] \\
\leq & \lambda^{-1} N \exp \left(-\frac{b^{2}}{2 \sigma_{T}^{2}}\right) \bar{Q}\left(\inf _{\delta_{0}<t \leq T} \int_{0}^{t} f(s) d s<\delta b\right) \\
= & \lambda^{-1} N \exp \left(-\frac{b^{2}}{2 \sigma_{T}^{2}}\right) E^{\bar{Q}}\left[\bar{Q}\left(\inf _{\delta_{0}<t \leq T} \int_{0}^{t} f(s) d s<\delta b \mid \iota\right)\right] .
\end{aligned}
$$

In the following we provide an upper bound of the expectation term in (20). Conditional on $\iota$, equation (19) implies that

$$
\int_{0}^{t} f(s) d s=\int_{0}^{t} C(s, \iota) b+g(s) d s .
$$

By condition $\mathrm{A} 3$, there exists a constant $\delta_{1}$ such that

$$
\inf _{\delta_{0}<t \leq T} \int_{0}^{t} f(s) d s \geq 2 \delta_{1} b+\inf _{\delta_{0}<t \leq T} \int_{0}^{t} g(s) d s .
$$


Now set $\delta$ in (18) equal to $\delta_{1}$ and we have

$$
\bar{Q}\left(\inf _{\delta_{0}<t \leq T} \int_{0}^{t} f(s) d s<\delta b\right) \leq \bar{Q}\left(\inf _{\delta_{0}<t \leq T} \int_{0}^{t} g(s) d s<-\delta b\right) .
$$

By the Borel-TIS lemma, there exists some constant $c>0$ such that

$$
\begin{aligned}
\bar{Q}\left(\inf _{\delta_{0}<t \leq T} \int_{0}^{t} g(s) d s<-\delta b\right) & \leq \bar{Q}\left(T\left|\sup _{t \in[0, T]} g(t)\right|>\delta b\right) \\
& \leq \exp \left(-c \delta^{2} b^{2}\right) .
\end{aligned}
$$

Together with (20), this implies that

$$
P\left(\inf _{\delta_{0}<t \leq T} \int_{0}^{t} f(s) d s<\delta b, \sup _{t} f(t)>b\right)=o(1) P\left(\sup _{t} f(t)>b\right) .
$$

Thus the first term in (18) is ignorable.

Consider the second term in (18). When $\inf _{\delta_{0}<t \leq T} \int_{0}^{t} f(s) d s>\delta b$, there exist $c, \delta^{*}>0$ such that for all $t \in\left[\delta_{0}, T\right]$,

$$
f(t)-c e^{-\delta^{*} b} \leq U(t)<f(t)
$$

Therefore, we have that

$$
\begin{aligned}
P\left(\sup _{0 \leq t \leq T} U(t)>b\right) & \geq P\left(\sup _{\delta_{0}<t \leq T} U(t)>b, \inf _{\delta_{0}<t \leq T} \int_{0}^{t} f(s) d s>\delta b, \sup _{t} f(t)>b, \mathcal{L}\right) \\
& \geq P\left(\sup _{\delta_{0}<t \leq T} f(t)>b+c e^{-\delta^{*} b}, \inf _{\delta_{0}<t \leq T} \int_{0}^{t} f(s) d s>\delta b, \sup _{t} f(t)>b, \mathcal{L}\right) .
\end{aligned}
$$

By (21) we have (22) is equal to

$$
\begin{aligned}
& P\left(\sup _{\delta_{0}<t \leq T} f(t)>b+c e^{-\delta^{*} b}\right)-o(1) P\left(\sup _{0<t \leq T} f(t)>b\right) \\
= & (1+o(1)) P\left(\sup _{\delta_{0}<t \leq T} f(t)>b\right)-o(1) P\left(\sup _{0<t \leq T} f(t)>b\right),
\end{aligned}
$$

where the equation follows from the density result in Lemma 7. Note that $\delta_{0}$ is chosen such that $\sup _{0 \leq t \leq \delta_{0}} \sigma(t)<\sup _{0 \leq t \leq T} \sigma^{2}(t)=\sigma_{T}$. The Borel-TIS lemma then indicates that

$$
P\left(\sup _{0 \leq t \leq \delta_{0}} f(t)>b\right)=o(1) P\left(\sup _{0 \leq t \leq T} f(t)>b\right) .
$$


Thus, (22)-(24) gives

$$
w(b) \geq(1+o(1)) P\left(\sup _{0 \leq t \leq T} f(t)>b\right) .
$$

Combining the result that $w(b) \leq P\left(\sup _{0 \leq t \leq T} f(t)>b\right)$ from Proposition 10, we have the first approximation result.

(ii). We proceed to prove the second conclusion. Note that

$$
\begin{aligned}
& P\left(b-c_{b}<\sup _{0 \leq t \leq T} U(t)<b+c_{b}, \mathcal{L}\right) \\
\leq & P\left(b-c_{b}<\sup _{\delta_{0} \leq t \leq T} U(t)<b+c_{b}, \mathcal{L}\right)+P\left(\sup _{t \leq \delta_{0}} f(t)>b-c_{b}\right) .
\end{aligned}
$$

By Borel-TIS lemma and Lemma 7, we know that for $b>\kappa_{1}|\log \eta|$ with $\kappa_{1}$ large enough,

$$
\begin{aligned}
P\left(\sup _{t \leq \delta_{0}} f(t)>b-c_{b}\right) & \leq \exp \left(-\frac{\left(b-c_{b}-E\left[\sup _{0 \leq t \leq \delta_{0}} f(t)\right]\right)^{2}}{2 \sup _{0 \leq t \leq \delta_{0}} \sigma^{2}(t)}\right) \\
& =o(1) 2 c_{b} \sigma_{T}^{-1}\left(b-c_{b}\right) P\left(\sup _{0 \leq t \leq T} f(t)>b-c_{b}\right) \\
& =o(1) 2 c_{b} F^{\prime}\left(b-c_{b}\right) .
\end{aligned}
$$

For the first term on the right-hand side of (25), we apply the same technique as in (18) that basically replace " $U(t)$ " by " $f(t)$ ". Thus, for $\kappa_{1}$ large enough and $b>\kappa_{1}|\log \eta|$, we have that

$$
P\left(b-c_{b}<\sup _{\delta_{0} \leq t \leq T} U(t)<b+c_{b}, \mathcal{L}\right) \leq(1+o(1)) P\left(b-c_{b}<\sup _{\delta_{0} \leq t \leq T} f(t)<b+c_{b}, \mathcal{L}\right) .
$$

Then apply Lemma 7 again and we have that

$$
\begin{aligned}
& P\left(b-c_{b}<\sup _{\delta_{0} \leq t \leq T} f(t)<b+c_{b}, \mathcal{L}\right) \\
\leq & (1+o(1)) 2 c_{b} \sigma_{T}^{-1}\left(b-c_{b}\right) P\left(\sup _{\delta_{0} \leq t \leq T} f(t)>b-c_{b}\right) \\
\leq & (1+o(1)) 2 c_{b} \sigma_{T}^{-1}\left(b-c_{b}\right) P\left(\sup _{0 \leq t \leq T} f(t)>b-c_{b}\right) \\
= & (1+o(1)) 2 c_{b} F^{\prime}\left(b-c_{b}\right) .
\end{aligned}
$$

This implies the existence of constant $c$ such that (15) holds. 


\section{Acknowledgement}

Guang Lin would like to thank the support by the Applied Mathematics Program within the Department of Energy (DOE) Office of Advanced Scientific Computing Research (ASCR) as part of the Collaboratory on Mathematics for Mesoscopic Modeling of Materials (CM4). PNNL is operated by Battelle for the U.S. Department of Energy under Contract DEAC05-76RL01830. Jingchen Liu is supported in part by NSF, CMMI-1069064 and NSF, SES-1323977.

\section{References}

Ablowitz, M. and Segur, H. (1981) Solitons and the Inverse Scattering Transform. SIAM, Philadelphia.

Adler, R., Blanchet, J. and Liu, J. (2012) Efficient Monte Carlo for large excursions of Gaussian random fields. Annals of Applied Probability, 22, 1167-1214.

Asmussen, S. and Glynn, P. (2007) Stochastic Simulation: Algorithms and Analysis. New York, NY, USA: Springer.

Asmussen, S. and Kroese, D. (2006) Improved algorithms for rare event simulation with heavy tails. Advances in Applied Probability, 38, 545-558.

Blanchet, J. and Glynn, P. (2008) Effcient rare event simulation for the maximum of heavy-tailed random walks. to appear in Annals of Applied Probability, 18, 1351-1378.

Blanchet, J., Leder, K. and Glynn, P. (2008) Strongly efficient algorithms for light-tailed random walks: An old folk song sung to a faster new tune. In $M C Q M C 2008$ (eds. P. LEcuyer and A. Owen), 227-248. Springer.

Blanchet, J. H. and Liu, J. (2008) State-dependent importance sampling for regularly varying random walks. Advances in Applied Probability, 40, 1104-1128.

Bogachev, V. (1998) Gaussian Measures. Mathematical Surveys and Monographs Volume 62. American Mathematical Society.

Borell, C. (1975) The Brunn-Minkowski inequality in Gauss space. Inventiones Mathematicae, 30, 207-216.

Calogero, F. and Degasperis, A. (1982) Solitons and the Spectral Transform I. North Holland, Amsterdam.

Debussche, A. and Printems, J. (1999) Numerical simulation of the stochastic Korteweg-de Vries equation. Physica D, 134, 200-226. 
Dodd, R., Eilbeck, J., Gibbon, J. and Morris, H. (1982) Solitons and Nonlinear Wave Equations. Academic Press, London.

Dudley, R. (1973) Sample functions of the Gaussian process. Ann. Probab., 1, 66-103.

Dupuis, P., Leder, K. and Wang, H. (2007) Importance sampling for sums of random variables with regularly varying tails. ACM Trans. Model. Comput. Simul., 17, 14.

Ehrhard, A. (1983) Symétrisation dans l'space de Gauss. Math. Scand., 53, 281-301.

Faddeev, L. and Takhtajan, L. (1987) Hamiltonian Methods in the Theory of Solitons. Springer-Verlag, Berlin.

Gardner, C., Greene, J., Kruskal, M. and Miura, R. (1967) Method for solving the Korteweg-de Vries equation. Phys. Rev. Lett., 19, 1095-1097.

Garnier, J. (1993) Long-Time Dynamics of Korteweg-de Vries Solitons Driven by Random Perturbations. Journal of Statistical Physics, 105, 789-833.

Garnier, J. (2001) Solitons in random media with long-range correlation. Waves Random Media, 11, 149-162.

Herman, R. L. (1990) The Stochastic, damped KdV equation. J. Phys. A: Math. Gen., 1063-1084.

Iizuka, T. (1993) Anomalous diffusion of solitons in random systems. Phys. Rev. Lett., 181, 39-42.

Konotop, V. and Vazquez, L. (1994) Nonlinear Random Waves. World Scientific, Singapore.

Lin, G., Grinberg, L. and Karniadakis, G. (2006) Numerical studies of the stochastic korteweg-de vries equation. J. Comp. Phys., 213, 676-703.

Liu, J. (2012) Tail approximations of integrals of Gaussian random fields. Annals of Probability, 40, 1069-1104.

Liu, J. and Xu, G. (2012a) On the density functions of integrals of Gaussian random fields. Advances in Applied Probability, 45, 398-424.

Liu, J. and Xu, G. (2012b) Some asymptotic results of Gaussian random fields with varying mean functions and the associated processes. Annals of Statistics, 40, 262-293.

Liu, J. and $\mathrm{Xu}$, G. (2013a) Efficient simulations for the exponential integrals of hölder continuous gaussian random fields. The ACM Transactions on Modeling and Computer Simulation, to appear. Available at http://stat.columbia.edu/ jcliu/paper/Tomacs3.pdf. 
Liu, J. and Xu, G. (2013b) On the conditional distributions and the efficient simulations of exponential integrals of Gaussian random fields. Annals of Applied Probability, to appear. Available at http://arxiv.org/abs/1204.5546.

Newell, A. (1985) Solitons in Mathematics and Physics. SIAM, Philadelphia.

Novikov, S., S.V. Manakov, L. P. and Zakharov, V. (1980) Theory of Solitons: Inverse Scattering Method. Consultants Bureau, New York.

Scalerandi, M. and Romano, A. (1998) Korteweg-de Vries equation under additive stochastic perturbations. Phys. Rev. E, 58, 4166-4173.

Tsirelson, B., Ibragimov, I. and Sudakov, V. (1976) Norms of Gaussian sample functions. Proceedings of the Third Japan-USSR Symposium on Probability Theory (Tashkent, 1975), 550, 20-41.

Wadati, M. (1983) Stochastic Korteweg-de Vries equation. J. of the Physical Society of Japan, 52, 2642-2648.

Wadati, M. and Akutsu, Y. (1984) Stochastic Korteweg-de Vries equation with and without damping. J. of the Physical Society of Japan, 53, 3342-3350.

Washimi, H. and Taniuti, T. (1966) Propagation of ion-acoustic solitary waves of small amplitude. Phys. Rev. Lett., 17, 996-998.

Zabusky, N. and Kruskal, M. (1965) Interaction of solitions in a collisionless plasma and the recurrence of initial states. Phys. Rev. Lett., 15, 240-243. 